Research Articles

\title{
Quantum Uncertainty and the Fifth Dimension
}

\author{
Paul S. Wesson \\ Department of Physics and Astronomy, University of Waterloo, Waterloo, Ontario N2L 3G1, Canada
}

Article history

Received: 06-01-2015

Revised: $30-01-2015$

Accepted: 30-01-2015

E-mail: psw.papers@yahoo.ca

\begin{abstract}
If the world has an extra dimension, the motion of a particle in spacetime may be slightly modified, producing a correlation between the momentum and position similar to that familiar from the uncertainty relation in quantum mechanics.
\end{abstract}

Keywords: Heisenberg's Relation, Extra Dimensions

\section{Introduction}

One of the most puzzling aspects of quantum mechanics is that it appears to be impossible to simultaneously know to arbitrary accuracy both the momentum and position of a particle. This uncertainity, usually associated with Heisenberg, involves a limit set by Planck's constant: $\Delta p \Delta x \sim h$. While familiar to every student of physics, this relation does not find a satisfactory explanation in conventional mechanics. However, it was pointed out some time ago that an explanation might in principle be available if the world has more than four dimensions (Wesson, 2002). Higher dimensions are currently popular as a means of unifying the interactions of physics. In particular, in five dimensions the laws of mechanics are perforce $5 \mathrm{D}$ rather than 4D in nature; and the difference can show up as a small perturbation which leads to an effect similar to quantum uncertainty. The aim of the present work is to take a new look at this subject, using results which have been found recently in 5D relativity.

One of the foundations of 5D relativity follows from an old embedding theorem of Campbell. Thus any 4D metric describing a vacuum in general relativity can be smoothly embedded in a 5D metric of so-called canonical type (Mashhoon et al., 1994; 1998). Recent work has focussed on more general types of embeddings (Romero et al., 1996; Dahia et al., 2003; 2008), cosmology and dynamics in 5D (Ponce de Leon, 1998; 2008; 2007; Liu and Mashhoon, 1995), the nature of inflation (Bellini, 2005; 2011) and astroparticle physics (Israelit, 2008; 2009). Of particular note is the application of the canonical metric to quantum mechanics (Wesson, 2011a; 2011b) and the discovery of a canonical solution to the 5D field equations which in 4D has the properties of a de Broglie wave (Wesson, 2013). Some results from these sources will be used below to help elucidate Heisenberg's uncertainty relation.
A relation of the form $\Delta p \Delta x \sim h$ can be seen to be peculiar even from the viewpoint of conventional $4 \mathrm{D}$ mechanics. For when it is divided by two increments in the proper time, it yields in the limit a statement to the effect that there is a force acting parallel to the velocity with a scalar product that is finite. This is contrary to the well-known orthogonality condition in 4D relativity, where the scalar product of the acceleration and the 4-velocity is zero. On the other hand, it will be shown in the next section that when $4 \mathrm{D}$ spacetime is extended to $5 \mathrm{D}$, an extra force (per unit mass) comes in which does indeed act parallel to the 4-velocity, thereby providing a possible explanation for the uncertainty relation. The technical analysis of section 2 is discussed in a more qualitative fashion in section 3 .

\section{D Dynamics and 4D Uncertainty}

For a test particle situated in the vacuum, it is natural to consider the canonical metric, because as noted above this is the $5 \mathrm{D}$ form which embeds all vacuum solutions of the 4D Einstein equations (Wesson, 2002; Mashhoon et al., 1994; 1998). In accordance with modern quantum field theory, the vacuum is regarded as an energetic medium with an energy density measured by the cosmological constant. These parameters can be positive or negative, depending on whether the extra dimension is spacelike or timelike (both are allowed in 5D relativity). The coordinates are $x^{A}=x^{a}, l$ for spacetime and the extra dimension $\left(A=0-4, a=0-3, \mathrm{x}^{4}=l\right)$. The fundamental constants will usually be absorbed to streamline the working, except where they are made explicit to aid physical understanding. Other aspects of the nomenclature are standard.

The 5D canonical metric in its simplest form can be written as: 


$$
d S^{2}=\left(\frac{l}{L}\right)^{2} \bar{g}_{\alpha \beta}\left(x^{\gamma}, l\right) d x^{\alpha} d x^{\beta}-d l^{2}
$$

Here $L$ is a constant length related to the 4D cosmological constant. The 4D interval is given by $d s^{2}=\bar{g}_{\alpha \beta} d x^{\alpha} d x^{\beta}$ and the metric of spacetime $\bar{g}_{\alpha \beta}\left(x^{\gamma}, l\right)$ can be used to normalize the 4-velocities $u^{\gamma} \equiv d x^{\gamma} / d s$ via $\bar{g}_{\alpha \beta} u^{\alpha} u^{\beta}=1$. Using this and employing the $4 \mathrm{D}$ proper time $s$ as affine parameter, it is straightforward to extremize the $5 \mathrm{D}$ interval (1). The result is a set of equations of motion for spacetime and a relation for the motion in the extra dimension Equation 2 and 3:

$$
\begin{aligned}
& \frac{d u^{\mu}}{d s}+\Gamma_{\beta \gamma}^{\mu} u^{\beta} u^{\gamma}=f^{\mu} \\
& f^{\mu} \equiv\left(-\bar{g}^{\mu a}+\frac{1}{2} \frac{d x^{\mu}}{d s} \frac{d x^{\alpha}}{d s}\right) \frac{d l}{d s} \frac{d x^{\beta}}{d s} \bar{g}_{\alpha \beta, 4} \\
& \frac{d^{2} l}{d s^{2}}-\frac{2}{l}\left(\frac{d l}{d s}\right)^{2} \\
& +\frac{l}{L^{2}}=-\frac{1}{2}\left[\frac{l^{2}}{L^{2}}-\left(\frac{d l}{d s}\right)^{2}\right] \frac{d x^{\alpha}}{d s} \frac{d x^{\beta}}{d s} \bar{g}_{\alpha \beta, 4}
\end{aligned}
$$

It is seen that in spacetime, conventional geodesic motion is perturbed by an extra force (per unit mass) or acceleration $f^{\mu}$, which is finite if there is motion in the extra dimension $(d l / d s \neq 0)$ and the 4D metric depends on the extra coordinate $\left(\bar{g}_{\alpha \beta},{ }_{4} \neq 0\right.$ where a comma denotes the partial derivative). These quantities also figure in the equation of motion for the extra dimension.

The extra acceleration in spacetime given by (2) is on inspection found to consist of a part $\left(f_{n}^{\mu}\right)$ normal to the 4-velocity $u^{\mu}$ and a part ( $\left.f_{p}^{\mu}\right)$ parallel to it. The normal component is akin to conventional forces and obeys the usual orthogonality condition $f_{n}^{\mu} u_{\mu}=0$. But the parallel component has no analog in $4 \mathrm{D}$ and is a unique indicator of an extra dimension with $f_{p}^{\mu} u_{\mu} \neq 0$. This parallel component can be written as Equation 4:

$$
f_{p}^{\mu}=-\frac{u^{\mu}}{2}\left(\bar{g}_{\alpha \beta}, u^{\alpha} u^{\beta}\right) \frac{d l}{d s}
$$

It is generally non-zero and since it depends on the relative velocity between the $4 \mathrm{D}$ and $5 \mathrm{D}$ frames it is inertial in the Einstein sense. But it is zero if there is no coupling between $4 \mathrm{D}$ and the fifth dimension $\left(\bar{g}_{\alpha \beta}, 4=0\right)$. This holds for what is sometimes called the purecanonical metric, where $\bar{g}_{\alpha \beta}=\bar{g}_{\alpha \beta}\left(x^{\gamma}\right.$ only $)$ and the extra coordinate enters only through the quadratic warp factor. It is important to realize, however, that this situation presumes that the 4-velocities are normalized without this factor, via $\bar{g}_{\alpha \beta} u^{\alpha} u^{\beta}=1$ (see above). The fact that the pure-canonical metric may show no deviation from conventional geodesic motion depends on a special choice of coordinates. It is therefore necessary to inquire further and ask about the generality of the force (4). This can be done by considering an unfactorized $l$-dependent metric with a general normalization condition on the 4velocities Equation 5:

$$
\begin{aligned}
& d S^{2}=g_{\alpha \beta}\left(x^{\gamma}, l\right) d x^{\alpha} d x^{\beta}-d l^{2} \\
& g_{\alpha \beta}\left(x^{\gamma}, l\right) u^{\alpha} u^{\beta}=1
\end{aligned}
$$

The second of these relations may be differentiated with respect to $s$ and a term expanded using the Christoffel symbols, to show that the motion again consists of the conventional geodesic with a perturbation. Remarkably, the latter has the same form as (4) above, but with $g_{\alpha \beta}\left(x^{\gamma}, l\right)$ in place of $\bar{g}_{\alpha \beta}\left(x^{\gamma}, l\right)$. This means that the fifth force (per unit mass) has the form (4) generally, regardless of the coordinate system being employed.

This raises the interesting possibility that the force (4) may have measurable effects in certain situations even if the metric is of canonical type. To investigate this, let us consider the canonical metric in the more general form where it has been applied to quantum mechanics (Wesson, 2011a; 2011b; Wesson, 2013). Thus a shift $l \rightarrow\left(l-l_{0}\right)$ is applied along the extra axis. The metric then has the form (5) with $g_{\alpha \beta}=\left(l-l_{0}\right)^{2} L^{-2} \bar{g}_{\alpha \beta}\left(x^{\gamma}\right)$, which can be written alternatively as Equation 6:

$d S^{2}=\left(\frac{l-l_{0}}{L}\right)^{2} d s^{2}+d l^{2}$

The extra coordinate is now taken to be timelike, which results in the presence of oscillations in the vacuum which have the same properties as de Broglie waves (Wesson, 2013). A detailed investigation shows that these waves have associated with them particles of mass $m$, specified by the Compton wavelength $L=h / m c$ (which is constant). The shift in (6) also causes the appearance of a hypersurface at $l=l_{0}$ where the energy density of the vacuum formally diverges. This can be called a membrane (though it has properties different from those of the $Z_{2}$ hypersurface in Membrane theory). The waves engendered by (6) travel along the membrane, following null geodesics with $d S^{2}=0$. This condition in (6) means that $d l /\left(l-l_{0}\right) \sim d s / L$, where for simplicity the sign choice associated with the reversibility of the motion in $x^{4}$ and the $\sqrt{-1}$ associated 
with the timelike extra dimension have both been dropped (they make no difference to the physics). It is important to realize that near the membrane, $d l /\left(l-l_{0}\right) \rightarrow 1$ necessarily, so $d s \rightarrow L$ also. Recalling that $L=h / m c$, the last relation shows that near the membrane there is quantization with $m c d s \sim h$.

The foregoing discussion of the shifted-canonical metric (6) can be combined with the previously derived expression for the extra force (4). The latter now reads $f_{p}^{\mu}=-\left(u^{\mu} / 2\right)\left(g_{\alpha \beta, 4} u^{\alpha} u^{\beta}\right)(d l / d s)$ where the 4-velocities are normalized via $g_{\alpha \beta} u^{\alpha} u^{\beta}=1$. Since $g_{\alpha \beta}$ is now proportional to $\left(l-l_{0}\right)^{2}$, the scalar coupling term is $g_{\alpha \beta, 4} u^{\alpha} u^{\beta}=2\left(l-l_{0}\right)^{-1}$ and the magnitude of the force is Equation 7:

$$
f_{p}^{\mu}=\frac{u^{\mu}}{\left(l-l_{0}\right)} \frac{d l}{d s}=\frac{u^{\mu}}{L}
$$

This can be rewritten as:

$$
\frac{d u^{\mu}}{d s}=\frac{1}{L} \frac{d x^{\mu}}{d s} \text { or } d u^{\mu}=\frac{d x^{\mu}}{L}
$$

Then we can form the scalar quantity $d u^{\mu} d x_{\mu}=d s^{2} / L$. This may in turn be re-expressed using the condition $d l /\left(l-l_{0}\right) \rightarrow 1$ wherein $d s / L \rightarrow 1$ (see above). The result is $d u^{\mu} d x_{\mu}=L$. Substituting for $L=h / m c$ from before and replacing the change in velocity by the change in momentum gives Equation 8:

$$
d p^{\mu} d x_{\mu}=h
$$

This is the same type of relation as the uncertainty limit typical of quantum mechanics.

\section{Discussion and Conclusion}

The analysis given above is admittedly fairly technical. However, it is also logical and involves a minimal extension of conventional mechanics. There are several approaches to higher-dimensional physics (Carr, 2007) and Membrane theory and Space-Time-Matter theory are notable in being in agreement with known data. The analysis of Section 2 follows the latter approach and it is instructive to summarize the derivation.

Given an extra dimension, it is natural that the laws of motion should be 5D rather than 4D in nature. Most particles are effectively situated in 4D vacuum and the most general 5D embedding for this is the shiftedcanonical metric. This leads to a model with waves as well as particles, concentrated around a kind of membrane caused by the shift along the extra axis. Unless the coordinates are specially chosen, this metric like others is characterized by an extra acceleration or force (per unit mass), which acts in spacetime. It is a signature of its $5 \mathrm{D}$ origin that this force acts parallel to the 4-velocity, unlike other 4D forces. Near the membrane, the metric obliges a relation between the increment in proper time and the Compton wavelength which leads to quantization. The same condition causes the change in the momentum and the change in the position of a particle to be correlated. This takes a form similar to what is familiar from quantum uncertainty.

That this is a reasonable result can be appreciated by expanding on a comment made previously. In $4 \mathrm{D}$, it is nearly always the case that the 4-velocities $u^{\alpha} \equiv d x^{\alpha} / d s$ of a massive particle are normalized via the condition $u^{\alpha} u_{\alpha}=1$. Differentiating this with respect to the $4 \mathrm{D}$ proper time $s$ and defining a force (per unit mass) $f^{\alpha} \equiv d u^{\alpha} / d s$ gives $f^{\alpha} u_{\alpha}=0$. This orthogonality condition is obeyed by known 4D forces, such as the pressure due to a perfect fluid or the electrodynamic force on a moving charge. However, the situation is different if there is another dimension. Then the corresponding normalization condition on the velocities is $u^{A} u_{A}=$ constant $(A=0-4)$, which differentiated gives $f^{\alpha} u_{\alpha}=-f^{4} u_{4} \neq 0$. That is, unless the coordinates are specially chosen, the 4D orthogonality condition is broken by the fifth dimension.

If quantum uncertainty is in fact due to an extra dimension, further investigations of 4D dynamics can be made using the approach outlined here. Perhaps the most obvious subject for further work concerns virtual particles, which are inferred to exist but cannot be observed directly due to the nature of their interactions. However, virtual particles are believed to lead to certain effects which are observed, such as vacuum polarization and the energy/range dependence of the fine-structure 'constant'. It would be interesting to re-examine such effects using $5 \mathrm{D}$ relativity.

\section{Acknowledgement}

This study is based on earlier work done by members of the Space-Time-Matter group (5Dstm.org).

\section{Ethics}

This article is original and contains unpublished material. The corresponding author confirms that the author have read and approved the manuscript and no ethical issues involved.

\section{References}

Bellini, M., 2005. De Sitter inflationary expansion from a noncompact KK theory: A nonperturbative quantum (scalar) field formalism. Phys. Lett. B, 609: 187-193. DOI: 10.1016/j.physletb.2005.01.056 
Bellini, M., 2011. Pre-big bang collapsing universe from modern Kaluza-Klein theory of gravity. Phys. Lett. B, 705: 283-286. DOI: 10.1016/j.physletb.2011.10.037

Carr, B.J., 2007. Universe or Multiverse? 1st Edn., Cambridge University Press, Cambridge, ISBN-10: 0521848415, pp: 517.

Dahia, F., C. Romero, L.F.P. da Silva and R. Tavakol, 2008. Geodesic motion in the neighbourhood of submanifolds embedded in warped product spaces. Gen. Rel. Grav., 40: 1341-1351. DOI: $10.1007 / \mathrm{s} 10714-008-0611-y$

Dahia, F., E.M. Monte and C. Romero, 2003. Fifth force from fifth dimension: A comparison between two different approaches. Mod. Phys. Lett. A, 18: 1773-1773. DOI: 10.1142/S0217732303011484

Israelit, M., 2008. Creation of fundamental particles in Wesson's IMT. Gen. Rel. Grav., 40: 2469-2492. DOI: $10.1007 / \mathrm{s} 10714-008-0633-5$

Israelit, M., 2009. Dark matter and cosmic acceleration from Wesson's IMT. Gen. Rel. Grav., 41: 2847-2866. DOI: $10.1007 / \mathrm{s} 10714-009-0811-0$

Liu, H. and B. Mashhoon, 1995. A Machian interpretation of the cosmological constant. Ann. Phys., 507: 565-582. DOI: 10.1002/andp.19955070604

Mashhoon, B., H. Liu and P.S. Wesson, 1994. Particle masses and the cosmological constant in KaluzaKlein theory. Phys. Lett. B, 331: 305-312. DOI: 10.1016/0370-2693(94)91057-X

Mashhoon, B., P.S. Wesson and H. Liu, 1998. Dynamics in Kaluza-Klein gravity and a fifth force. Gen. Rel. Grav., 30: 555-571. DOI: 10.1023/A:1018814123514
Ponce de Leon, J., 1988. Cosmological models in a Kaluza-Klein theory with variable rest mass. Gen. Rel. Grav., 20: 539-550. DOI: 10.1007/BF00758909

Ponce de Leon, J., 2007. Embeddings for 4D Einstein equations with a cosmological constant. Grav. Cosmol., 14: 241-247. DOI: $10.1134 / \mathrm{S} 0202289308030067$

Ponce de Leon, J., 2008. The principle of least action for test particles in a four-dimensional space-time embedded in 5D. Phys. Lett. A, 23: 249-249. DOI: $10.1142 / \mathrm{S} 0217732308026376$

Romero, C., R. Tavakol and R. Zalaletdinov, 1996. The embedding of general relativity in five dimensions. Gen. Rel. Grav., 28: 365-37. DOI: $10.1007 / \mathrm{BF} 02106973$

Wesson, P.S., 2002. Classical and quantized aspects of dynamics in five-dimensional relativity. Class. Quant. Grav., 19: 2825-2825. DOI: $10.1088 / 0264-9381 / 19 / 11 / 306$

Wesson, P.S., 2011a. General relativity and quantum mechanics in five dimensions. Phys. Lett. B, 701: 379-383. DOI: 10.1016/j.physletb.2011.05.038

Wesson, P.S., 2011b. The cosmological 'constant' and quantization in five dimensions. Phys. Lett. B, 706: 1-5. DOI: 10.1016/j.physletb.2011.10.027

Wesson, P.S., 2013. Vacuum waves. Phys. Lett. B, 722: 1-4. DOI: 10.1016/j.physletb.2013.04.005 